\title{
LA POLÍTICA EN LAS CALLES APROXIMACIONES DESDE LA ARGENTINA RECIENTE
}

\section{Julián Rebón}

\section{Resumen}

La Argentina reciente, como lo indican diversos estudios, se caracteriza por una significativa dinámica de acción colectiva. Este trabajo se interroga sobre el impacto político de la protesta social en el país durante el período 2001-2017. Propone como hipótesis central que la protesta representa un recurso político relevante. Esta participa de la disputa general por la direccionalidad del país, varía en su composición en las distintas etapas del período y tiene un impacto predominantemente de carácter negativo sobre la acción de las autoridades estatales. El desarrollo de las hipótesis se basa en una perspectiva panorámica que da cuenta de los principales componentes de la protesta en su contexto histórico. El artículo sistematiza diversos avances investigativos, propios y de otros equipos.

Palabras clave: protesta social / Argentina / acción colectiva / impacto político.

\section{Abstract}

\section{Politics on streets. Approaches from recent Argentina}

The recent Argentinian history presents a significant dynamic of collective action. This paper asks about the political impact of social protest in the country during the period 2001-2017. It proposes as a central hypothesis that protest is a relevant political resource. It participates in the general dispute over the direction of the country, varying in its composition in the different stages of the period and having a predominantly negative impact on the action of state authorities. The development of the hypothesis presents a panoramic perspective that accounts for the main components of the protest in its historical context. The sources of the work come from the systematization of research of different teams in the field.

Keywords: social protest / Argentina / collective action / political impact.

Julián Rebón: Doctor en Ciencias Sociales. Profesor titular de la Universidad de Buenos Aires. Instituto de Investigaciones Gino Germani, Facultad de Ciencias Sociales, Universidad de Buenos Aires, Consejo Nacional de Investigaciones Científicas y Técnicas (Argentina).

E-mail: julianrebon@gmail.com 


\section{Introducción ${ }^{1}$}

La vida política contemporánea de Argentina está signada por el período más largo de funcionamiento del régimen democrático-constitucional. A diferencia de lo ocurrido en el pasado, las confrontaciones políticas tienden a ser resueltas en este marco y la democracia institucional como forma de gobierno es el horizonte en el cual se inscribe el conjunto de los actores políticos. Paradójicamente, el consenso ciudadano en torno a la democracia -como proyecto - implica marcados cuestionamientos de diversos tipos a su funcionamiento real. Así como en otros países de la región y el mundo, se registran altos niveles de desconfianza en los partidos y los políticos (Rosanvallon, 2007; Cheresky, 2011). La relación entre ciudadanía y sistema político desborda ampliamente la votación de sus representantes, y se desarrolla un conjunto de mecanismos de institucionalidad diversa y actuación recurrente que implica elementos de control, participación, juzgamiento y limitación de los gobernantes en su accionar cotidiano. En este marco, la protesta social destaca como un recurso político de carácter informal que canaliza demandas a las autoridades, veta o bloquea acciones de gobierno, incorpora temas en la agenda pública y configura un renovado espacio de participación ciudadana (Moscovich, et al., 2017; Schuster, 2011; Svampa, 2017).

Históricamente, la acción colectiva y la protesta han tenido un lugar destacado en la historia del país (Lobato y Suriano, 2003). En la última parte del siglo XX, en condiciones de deterioro de los indicadores sociales y de heterogeneización de las clases subalternas, se produjo una diversificación de los actores y repertorios de la acción colectiva (Auyero, 2002; Pereyra, et al., 2017). A inicios del siglo XXI, la protesta se había convertido en un recurso político significativo de contenido diverso (Schuster, 2011; Gómez, 2008). Este trabajo se propone profundizar y especificar el impacto político de la protesta a partir del análisis de sus principales componentes durante las distintas etapas del período 2001-2017. En este marco nos interrogamos: ¿En qué ha consistido y cómo ha evolucionado durante lo que va del siglo XXI el impacto de la protesta social sobre la acción de las autoridades del Estado? ¿Cuáles han sido sus mecanismos y contenidos

1 Este artículo fue escrito en el marco de dos estadías de investigación en la Universidad de Calabria y en la Universidad de Tampere, financiadas por el proyecto INCASI de Investigación e Innovación Horizonte 2020 de la Unión Europea (MSCA, GA-691004). 
centrales? ¿Qué formas y composiciones sociales y políticas asume principalmente la protesta en cada etapa?

El período propuesto es particularmente rico para los interrogantes planteados, dado que registra transformaciones en las dimensiones centrales para el estudio de la protesta, las condiciones políticas (Tilly, 2008) y los sistemas de acumulación e intercambio (Silver, 2005). La periodización de la evolución reciente de la Argentina en estos términos identifica tres etapas que nos muestran un territorio en disputa entre fuerzas sociales diversas, cuya direccionalidad no se encuentra consolidada. En tiempos de crisis refiere al período signado por la crisis general de 2001, que expresa el derrumbe de la hegemonía del modelo de valorización financiera consolidado a partir de las reformas neoliberales de los noventa. Involucra en su desarrollo la renuncia del presidente Fernando de la Rúa (1999-2000, Unión Cívica RadicalAlianza $^{2}$ ) en diciembre de dicho año y se prolonga con nitidez hasta 2003 , con la asunción de un nuevo gobierno electo. La segunda etapa, En tiempos de kirchnerismo, refiere al ciclo de gobierno iniciado por la presidencia de Néstor Kirchner (2003-2007), del Partido Justicialista (PJ) y Frente para la Victoria $\left(\mathrm{FPV}^{3}\right)$, continuado luego por Cristina Fernández de Kirchner (PJFPV, 2007-2015). En este ciclo se recompone el orden social y se plantea una relación crítica con la herencia neoliberal, que alcanza incluso a alterar tendencias sociales y económicas dominantes hasta entonces. Finalmente, con En tiempos de Cambiemos nos referimos al período abierto a partir de la asunción de Mauricio Macri (PRO-Cambiemos ${ }^{4}$, 2015-actualidad) que, haciendo honor al nombre de la coalición electoral, está produciendo una política contrastante con el ciclo previo, retomando la agenda de reformas neoliberales.

El presente artículo postula como hipótesis central que la protesta social, entendida como episodio de acción colectiva contenciosa de reclamo público a las autoridades, representa un recurso político relevante a lo largo del período. La protesta social participa de la disputa general por la direccionalidad del país, variando en su composición social en las distintas etapas y teniendo un impacto predominantemente negativo. Con el objeto

2 La Alianza fue una coalición entre la Unión Cívica Radical (UCR) — partido centenario con base en las capas medias, signado en su origen por una orientación reformista y republicanay distintos sectores de centroizquierda.

3 El PJ es la principal expresión partidaria del peronismo, movimiento reformista de orientación nacional y popular y composición policlasista - con fuerte base en los trabajadores y pobres urbanos - surgido a mediados del siglo XX. El FPV representa en el período un frente electoral de centroizquierda liderado por el PJ, con distintos aliados menores.

$4 \quad$ El PRO es un partido de reciente formación con una orientación política de centro-derecha y origen en el empresariado. Actualmente, lidera la coalición Cambiemos, de la cual también participan la UCR y otros aliados. 
de desarrollar las hipótesis, utilizamos una perspectiva panorámica que da cuenta de los principales componentes de la protesta social en su vinculación con el contexto en el cual se desarrollan y en la sucesión histórica de las etapas.

El trabajo no pretende hacer un recorrido exhaustivo de la protesta en cada etapa. Más bien procura identificar qué componentes de la acción colectiva, entendida en términos de Tilly (2008) como acción grupal discontinua y contenciosa, son los centrales en la confrontación política en cada período. En este sentido, el criterio de selección de las acciones y los actores utilizado es su masividad e impacto en la direccionalidad política del país. El artículo sistematiza diversos avances investigativos, propios y de otros equipos. También, cuando la argumentación lo requiere, recurre al desarrollo narrativo de acontecimientos o la presentación de datos primarios o secundarios. El enfoque teórico parte de una perspectiva heterodoxa que integra a diferentes autores en función del objeto de estudio. En este sentido, en el texto se presentan referencias teóricas vinculadas tanto a la teoría de los procesos políticos en el campo de la acción colectiva (Tilly, 2008), como a las perspectivas clasistas del análisis social (Marín, 2009; Silver, 2005; Wright, 2000).

Finalmente, corresponde señalar que el artículo se estructura a partir de la periodización planteada y concluye con reflexiones que sistematizan los hallazgos encontrados y propone desafíos pendientes para la investigación y la acción.

\section{En tiempos de crisis}

El siglo XXI, en términos históricos (Hobsbawm, 1997), comenzó en Argentina con la denominada crisis de 2001. Para comienzos de dicho año eran notorios los signos de agotamiento del modelo de acumulación estructurado sobre las reformas neoliberales de los noventa ${ }^{5}$. Destacaban el creciente peso en la economía de la deuda externa y del déficit fiscal, la falta de competitividad de la producción local y el inusitado aumento del desempleo y la pobreza. El ajuste del modelo llevado adelante por el gobierno de De la Rúa, lejos de a los efectos deseados, condujo al estancamiento económico y a la dificultad creciente para conseguir financiamiento a tasas razonables. En este marco, creció la resistencia a los procesos de expropiación de derechos y de exclusión social por parte de sindicatos y del movimiento de trabajadores desocupados (conocidos como piqueteros) con acciones cada vez más disruptivas y metas más politizadas (Gómez, 2009). En octubre, el gobierno consolidó el modelo de acumulación con eje en la valorización financiera (Basualdo, 2013). 
perdió las elecciones parlamentarias de medio término. La disconformidad ciudadana no se redujo a votar a las alternativas opositoras, sino que incluyó el uso no convencional de las instituciones: el creciente malestar con los partidos políticos llevó a que el voto en blanco y el anulado alcanzaran a un cuarto del electorado, un porcentaje inédito para la historia reciente (Pucciarelli y Castellani, 2014).

Diciembre fue el mes en el cual se desató la crisis general, de carácter orgánico, en la que la perturbación del ordenamiento social en sus distintos ámbitos condujo al colapso de la hegemonía neoliberal (Pierbattisti, 2017; Pucciarelli y Castellani, 2014). El mecanismo constitutivo de la crisis no puede reducirse a lo económico; la acción de resistencia de las distintas identidades que ven alteradas sus condiciones de vida - dislocación social- es central para entender su desarrollo. Esta dislocación social, como es habitual en el marco de procesos de mercantilización (Polanyi, 2001), ocasionó resistencias en múltiples sectores de la estructura social. Las medidas económicas impulsadas por el ministro de Economía Domingo Cavallo - también autor de las reformas de los noventa - precipitaron el final del gobierno. El establecimiento del "corralito", con el cual se limitó el uso libre de los depósitos bancarios, desencadenó la profundización de la protesta social contra las políticas de gobierno y la realización de una huelga general el 13 de diciembre, con alto acatamiento, convocada por el conjunto del sindicalismo ${ }^{6}$. La huelga se transformó en la práctica en un paro activo que incluyó el apoyo de otros sectores de la población, tales como comerciantes o grupos piqueteros (Lobato y Suriano, 2003; Merino, 2012).

En los días posteriores, la rebelión social y la descomposición del orden se expresaron bajo diversas formas a lo largo y ancho del país. En este contexto, emergió y se difundió una forma de acción colectiva que, sin expresar un contenido nítidamente político, tendría significativas consecuencias en este campo. Nos referimos al desarrollo de saqueos a supermercados y comercios minoristas, primero en centros urbanos del interior y luego en la propia Área Metropolitana de Buenos Aires (AMBA), que en ocasiones

6 Las reformas de los noventa implicaron un retroceso para el poder estructural (Wrigth, 2000) de los trabajadores. No obstante, el poder de los sindicatos y los recursos de sus cúpulas no fueron debilitados en la misma medida (Etchemendy y Collier, 2008). La estrategia adaptativa del sindicalismo fue exitosa en preservar el modelo sindical con sus prerrogativas. La estrategia sindical de corte participacionista abrió espacio a la emergencia de otros nucleamientos que enfrentaron la reestructuración. Desde el corazón del sindicalismo peronista, en el marco de la histórica Central General de Trabajadores (CGT) y del modelo sindical tradicional, se conformó el Movimiento de los Trabajadores Argentinos encabezado por el sindicalista camionero Hugo Moyano. Por otra parte, se creó la Central de Trabajadores Argentinos (CTA). Esta central reivindicó un nuevo modelo sindical que plantea el pluralismo organizativo y la afiliación de los trabajadores no registrados. $\mathrm{Su}$ relevancia fue significativa entre los trabajadores estatales y organizaciones sociales, pero en ningún momento logró alterar la primacía de la CGT (Merino, 2012). 
devinieron en violentos enfrentamientos con las fuerzas del orden y los comerciantes (Auyero, 2007; Iñigo Carrera y Cotarelo, 2006). Su generalización, en el clima de protesta social ya reseñado, condujo al presidente, en la noche del 19 de diciembre, a declarar el estado de sitio como mecanismo de contención de la acción colectiva. Lejos del resultado esperado, emergió súbitamente la desobediencia civil generalizada. Con eje en las capas medias de la población, la base social originaria del gobierno, en diversos barrios de la ciudad de Buenos Aires el discurso del presidente anunciando el estado de sitio fue respondido con cacerolazos y manifestaciones espontáneas de carácter masivo que rápidamente inundaron los barrios de la ciudad para luego movilizarse hacia los centros del poder político. Las movilizaciones abarcaron diversas ciudades del país.

La inédita irrupción de masas encontró en el hacer ruido y en la bandera argentina los símbolos primarios de la protesta ciudadana. El desafío a la disposición presidencial entrañó con nitidez un reclamo destituyente hacia las personificaciones centrales de la política institucional: "Que se vayan todos, que no quede ni uno solo" se transformó rápidamente en la consigna de las movilizaciones del período. La movilización pacífica devino esa madrugada en incidentes ante la represión policial. El día 20, a pesar de la renuncia del ministro de Economía, las movilizaciones continuaron, esta vez convocadas por organizaciones sociales y políticas. La fuerte represión policial que intentaba despejar las inmediaciones de la casa de gobierno fue respondida por grupos de manifestantes no dispuestos a ceder en su determinación de lucha. Así, emergieron amplios y dilatados combates callejeros, ataques a edificios públicos, empresas (en particular bancos extranjeros) y algunos saqueos a negocios que caotizaron el área céntrica de la principal ciudad del país ${ }^{7}$.

Por la tarde, el PJ, principal fuerza de la oposición, rechazó la convocatoria presidencial a un gobierno de unidad ante la situación de crisis y el presidente se vio forzado a renunciar por la falta de apoyo y legitimidad para continuar en el cargo. El justicialismo impuso los términos de la sucesión. Primero, el Parlamento designó a Adolfo Rodríguez Saá (PJ), quien a los pocos días debió renunciar, en el marco de una protesta que terminó con incidentes frente a la casa de gobierno, ante la falta de apoyo de los gobernadores justicialistas. Poco después asumió interinamente la presidencia Eduardo Duhalde, senador del PJ y excandidato presidencial derrotado por De la Rúa en 1999.

7 Entre el 19 y el 20 de diciembre se produjo el asesinato de treinta y ocho personas en el contexto de la represión de la acción colectiva, la inmensa mayoría a manos de la fuerza de seguridad. La mayor parte de los casos se produjo en los barrios vinculados a situaciones de saqueos. La no existencia de muertos en las fuerzas de seguridad nos marca claramente la asimetría en las condiciones de la violencia. La mayoría de los homicidios continúan judicialmente impunes (La Vaca, 2011). 
El estado de ánimo ciudadano se prolongó en los meses subsiguientes. Se vivía en las calles una situación de ebullición y movilización social. Se formaron asambleas barriales que procuraban prolongar la indignación ciudadana del "19 y 20" en términos de cambio social y político (Svampa, 2017). Ahorristas afectados por el "corralito" atacaron los bancos reclamando la devolución de sus depósitos. Trabajadores desobedeciendo el desempleo asumieron colectivamente el mando de las empresas en crisis que los empleaban (Rebón, 2007). También fue una etapa en la que el movimiento piquetero surgido a fines de los noventa se consolidó y ganó en su capacidad de movilización (Maneiro, 2012), al superar en magnitud de conflictividad durante 2002 y 2003 al actor sindical (datos de PIMSA citados en Antón, et al., 2011), que merma su protagonismo producto de la integración de sectores sindicales al gobierno y de las adversas condiciones del mercado de trabajo para los asalariados. En un contexto marcado por la politización y la estructuración de solidaridades entre los movilizados, las organizaciones piqueteras encontraron más recepción pública a su protesta plebeya, en especial por parte de los sectores medios activados (Svampa, 2017). El período estuvo signado por la autonomización, por la crisis de las heteronomías preexistentes y la desobediencia anticipada a la autoridad en distintos grupos sociales (Rebón, 2007). Fue el tiempo de la asamblea y la democracia directa en las calles, de los debates acerca del cambio social, de la crítica al Estado y de la creencia en la potencia de la sociedad civil. Fue el momento de la acción directa, del desborde de los canales institucionales de procesamiento del conflicto, del piquete en la vía pública, de la toma de edificios y fábricas, del ataque a bancos. Fue la etapa de la experimentación en la búsqueda de formas alternativas de producción de condiciones de vida, como emprendimientos productivos autogestionados, huertas comunitarias y clubes de trueque. Fueron los tiempos de experimentar en el campo de la cultura, de los acontecimientos estético-políticos en el espacio público, de la formación de bachilleratos populares y centros culturales (Svampa, 2017).

La protesta social y la autonomización plantearon un fuerte desafío al gobierno provisional. Este focalizó su acción en una agenda de recomposición del orden que incluyó medidas heterodoxas. A pesar de materializar una brutal transferencia regresiva de ingresos para los sectores populares, a partir de la devaluación de la moneda y otros mecanismos, desarrolló, en simultáneo, la ampliación de compensaciones sociales, como el congelamiento de las tarifas de los servicios públicos, la expansión de la política social y el otorgamiento de reconocimientos selectivos a los actores de la protesta. Estos reconocimientos no alcanzaron a aquellos sectores que prolongaron su movilización; para estos, la respuesta fue crecientemente la confrontación. El asesinato de dos militantes y las decenas de heridos en el marco de la represión a una protesta piquetera que procuraba cortar el puente Pueyrredón 
el 26 de junio de 2002 fue la expresión más acabada de este intento represivo por contener la protesta. La ola de indignación y protesta desatada llevó a un cambio de estrategia. Se ampliaron la política de reconocimiento a las organizaciones sociales y las concesiones a los sectores populares, y se convocó a elecciones como salida ante la crisis de legitimidad (Gómez, 2009). Con estas elecciones se consagraría presidente Néstor Kirchner.

Los tiempos de crisis representan un punto de llegada y de acumulación de las resistencias que se venían produciendo desde fines de los noventa y un punto de encuentro con nuevos sectores que se activaron, configurando la protesta desde múltiples posiciones de la estructura social. La protesta no fue solo la expresión de la crisis, fue también un mecanismo de su estructuración. Al resistir los procesos de expropiación y exclusión, al impugnar el ordenamiento político, limitó la viabilidad de las políticas a llevar a cabo y construyó el escenario que precipitó la caída del gobierno de De la Rúa. Si bien otras formas de la política $-\mathrm{y}$ otros actores - fueron determinantes en dicha caída y en la transición abierta a partir de ella, la forma que esta asumió no puede explicarse ni entenderse sin la presencia de la acción colectiva en las calles ${ }^{8}$. Esta acción colectiva configuró una forma de política salvaje (Tapia, 2008) que limitó y bloqueó la acción de gobierno. Su contenido a nivel macro fue más destituyente que instituyente: expresó capacidad de veto más que de construcción de una direccionalidad política del país. Su poder instituyente se focalizó en diferentes espacios a nivel meso y micro, en los cuales se desenvolvió un rico proceso de autonomización. Los movilizados no lograron articular una dualidad de poder. Con el paso del tiempo, se fueron desgajando, dividiendo y, en ocasiones, desactivando, con distintas temporalidades. Sin embargo, los acontecimientos de los tiempos de crisis dejaron profundas huellas en la Argentina por venir. Ampliaron el repertorio de confrontación de diferentes grupos sociales y alteraron el balance entre formas de lucha prescriptas, toleradas y proscriptas por el Estado al incrementar el espacio de tolerancia. Conformaron nuevas culturas de lucha con una fuerte predisposición a la acción directa y a la desobediencia a la autoridad (Rebon, 2007; Svampa, 2017) que se activaron y recrearon a posteriori. Pero, sobre todo, dejaron latente en la memoria colectiva de la sociedad civil y de las personificaciones del Estado que el humo de la política en las calles siempre puede volver. La acción colectiva quedó así anclada como horizonte de amenaza destituyente.

8 Incluso actores de la política institucional incidieron en la promoción de acciones colectivas, como por ejemplo en los saqueos (Auyero, 2007). 


\section{En tiempos de kirchnerismo}

El ciclo kirchnerista, más allá de las características políticas y los rendimientos sociales diferenciados entre sus distintos momentos (Kulfas, 2016), estuvo signado en su conjunto por una impronta crítica de las tendencias dominantes en el período previo, que lo sitúa en el amplio abanico de las experiencias de gobiernos progresistas latinoamericanos (González, et al., 2010).

El origen del gobierno de Kirchner estuvo marcado por la crisis general de 2001. Por una parte, porque la posibilidad de su existencia se debió a ella. Fue en el contexto de impugnación de las personificaciones políticas dominantes que el por entonces gobernador de Santa Cruz, perteneciente a un sector periférico del PJ, logró construir su candidatura política. Por otra parte, porque su gobierno respondió a la demanda generalizada y con múltiples componentes de reconstrucción del orden (Piva, 2015). En la respuesta a esta demanda retomó selectivamente los reclamos de las luchas sociales del período previo, planteando una agenda reformista con distintas intensidades y temporalidades según los campos (justicia, internacional, derechos humanos, laboral, económico, entre otros). Se planteó como una recomposición reformista del orden, de corte más pragmático que ideológico, en la cual la política ganó márgenes de autonomía con respecto al poder económico, aprovechando las condiciones de oportunidad abiertas. Más que reflejar la correlación de fuerzas en la sociedad civil, utilizó la iniciativa estatal para transformarla. Sin una articulación orgánica con la cúspide social, la agenda de cambio fue el mecanismo de construcción de legitimidad pública y fuerza propia, dada la debilidad de origen ${ }^{9}$. Frente al fracaso de la política represiva sobre la protesta desarrollada por De la Rúa y Duhalde, el nuevo gobierno privilegió el diálogo con las organizaciones. Su construcción política de corte movimientista (Natalucci, 2017) y populista (Laclau, 2005) promovió apertura y reconocimiento hacia los actores movilizados, impulsando concesiones activas a sus demandas. Focalicémonos en el actor sindical por su centralidad en la coalición y en la conflictividad del período.

La política de Kirchner profundizó la recuperación económica evidenciada en los últimos meses del gobierno de Duhalde, promoviendo la producción industrial y el mercado interno. La fuerte expansión del empleo asalariado y la rápida baja del desempleo incrementaron el poder estructural de los trabajadores en el mercado de trabajo. El crecimiento del empleo, registrado en especial en ramas con fuerte presencia sindical, y los cambios en la legislación laboral favorecieron el poder institucional de los sindicatos (Et-

9 Recordemos que Kirchner asumió la presidencia ante el retiro del otro contrincante del balotaje, luego de haber salido segundo y sin contar con vínculos significativos con las principales organizaciones gremiales y sociales. 
chemendy, 2013). Estos encontraron condiciones para un rápido crecimiento en afiliados y recursos. Se produjo una "revitalización sindical", como suele denominarse en la literatura especializada al creciente peso de estas organizaciones, la difusión de las comisiones internas en el lugar de trabajo, el crecimiento exponencial de la firma de convenios colectivos y el renovado conflicto laboral (Etchemendy, 2013; Palomino y Dalle, 2016; Varela, 2017). En un marco en el que los actores más dinámicos del período previo tendieron a desaparecer - las asambleas barriales, por ejemplo- o a paulatinamente desmovilizarse y reformularse - como los piqueteros -, los trabajadores ocupados volvieron a representar a partir de 2004 el principal componente social del conflicto (datos de PIMSA citados en Antón, et al., 2011). La rápida recuperación salarial, en particular para los trabajadores bajo convenio del sector privado, retroalimentó el mercado interno y el crecimiento económico. Los conflictos fueron mutando desde las estrategias defensivas de la etapa previa, vinculadas a la preservación de la fuente laboral y los salarios adeudados, hacia metas ofensivas que implicaron el aumento del salario real y la mejora de las condiciones de trabajo y contratación, como por ejemplo la emergencia de luchas contra la precarización laboral. De enfrentar procesos expropiatorios, el conflicto laboral pasó al terreno de las condiciones de explotación de la fuerza de trabajo. Por supuesto, estas tendencias sufrieron alteraciones en el período, principalmente según los ciclos económicos. Pero, en línea generales, la interacción entre política de gobierno y conflicto laboral produjo procesos de movilidad social colectiva que transformaron las fronteras entre clases. La lucha corporativa de los trabajadores estables en las condiciones políticas descriptas no solo mejoró sus condiciones de vida, sino que logró también una movilidad colectiva ascendente que alteró la estructura de clase (Palomino y Dalle, 2016). Este proceso formó parte de una tendencia general en el período - no exenta de contradicciones en sus distintos ámbitos - a una mayor igualación social (Kessler, 2014).

Así se configuró una dinámica relacional convergente entre gobierno y sindicalismo. Este último - encarnado en los sectores mayoritarios de ambas centrales - fue un integrante fundamental de la coalición política. Este actor limitó el carácter político del conflicto laboral y se comprometió en la coordinación de salarios con el gobierno a través de paritarias de gremios testigo o instituciones colectivas como el Consejo del Salario. También participó a través de la movilización de sus bases en apoyo al gobierno, en particular en situaciones de conflicto de este con otros actores. Esta política de articulación de intereses estableció un patrón de acuerdo corporativista de carácter segmentado - expresión de los límites de la representación sindical existente-, en el cual los sindicatos operaron como un actor que intercambiaba la moderación del conflicto laboral y el poder de movilización por compensaciones organizativas y políticas para las entidades gremiales y salariales de 
los trabajadores bajo convenio (Etchemendy, 2013). De este modo, la incidencia política del sindicalismo no transitó por el andarivel de la protesta social contra el gobierno. Por el contrario, su dimensión política provino más bien de su alianza y movilización de apoyo hacia él.

No obstante, esta coalición encontró límites ${ }^{10}$ que se fueron profundizando con el paso del tiempo, tanto por obstáculos políticos como por limitantes estructurales del modelo de acumulación. A partir de 2011 emergió, desde el centro del sindicalismo peronista aliado al gobierno, el principal cuestionamiento, encabezado por Hugo Moyano, entonces secretario general de la CGT. Si bien un conjunto de condiciones organizativas y económicas formaron parte del contexto de esta ruptura y de los años subsiguientes, entre ellas la creciente dificultad para el aumento del salario real y la relevancia del impuesto a las ganancias para los trabajadores mejor pagos (Natalucci, 2017), el conflicto fue centralmente político. El empoderamiento del sindicalismo nutrió el anhelo de recuperar su peso político en el peronismo de otros momentos de la historia. Este proyecto se encontró con la oposición de la estructura territorial del peronismo y de la presidenta, decidida, luego de su reelección, a construir una fuerza más orgánica y con menos mediaciones con su base social (Etchemendy, 2013). La ruptura entre Moyano y el gobierno implicó la división de la CGT. A pesar de ser minoritaria, esta fracción produjo, en articulación táctica con otros sectores sindicales y sociales, las cinco huelgas generales — de acatamiento dispar - que se realizaron contra el gobierno durante toda la etapa. Los reclamos de paritarias libres y contra el impuesto a las ganancias enarbolados en los paros evidenciaron la crisis del pacto corporativo.

Como ya señalamos, la revitalización sindical implicó un mayor peso de este actor en la protesta y una institucionalización del conflicto social. Pero estas tendencias no pueden soslayar dos elementos centrales. En primer lugar, el carácter diverso de la protesta, alcanzado con nitidez en los noventa, muestra claras señales de continuidad (Pereyra, et. al., 2017). En el período, una multiplicidad de actores y situaciones fueron objeto de las acciones colectivas. El siempre presente movimiento de derechos humanos, los emergentes conflictos socioambientales, el renovado movimiento de las mujeres, la activación del movimiento de la economía popular, las luchas

10 Un ejemplo de estas limitaciones es la recurrencia de conflictos que no pudieron ser encauzados y regulados por el gobierno y, en ocasiones, por las cúpulas sindicales. El creciente poder estructural de los trabajadores y los procesos de recomposición de clase en su conjunto alimentaron la formación de comisiones internas y la emergencia de sindicalismo de base, en ocasiones con orientaciones clasistas, que produjeron conflictos con importante repercusión pública (Spaltemberg, 2012; Varela, 2017). Estos buscaron sin demasiado éxito romper el techo salarial y enfrentar a las conducciones sindicales, que persistieron con bajos niveles de renovación. 
contra la denominada inseguridad, los movimientos de los pueblos originarios, entre muchos otros, dan muestra del amplio abanico de la protesta social. En segundo lugar, la institucionalización de la acción colectiva, es decir, su canalización por las formas de acción prescriptas por la institución estatal, continuó siendo parcial.

Dos elementos colaboraron en tal dirección. En primer lugar, la continuidad de las culturas de lucha emergentes de 2001, signadas por la disrupción como forma de instalar socialmente el reclamo. En segundo lugar, la política represiva del Estado nacional que, en línea general, fue relativamente tolerante a dichas formas (Pérez y Rebón, 2012). En este marco, la acción directa, con los cortes como forma emblemática, persistió con relevancia en el repertorio de distintos grupos (Antón, et al., 2011; CENM, 2017) ${ }^{11}$. Estas formas tendieron a tener mayor uso por aquellos actores con menor poder estructural e institucional, con clara dificultad para obtener respuestas a sus reclamos por los canales institucionales (Rebón y Hernández, 2017), pero, también, como veremos posteriormente, por aquellos que, habiendo perdido posiciones en el campo estatal para realizar sus metas, tuvieron que pasar del lobby a la protesta en el espacio público.

La política del gobierno fue estructurando en sus confrontaciones un proceso de polarización política. La acción colectiva y los movimientos populares no fueron ajenos al clivaje político en torno al alineamiento frente al gobierno. Desde el inicio, la política de apertura del gobierno promovió la división del campo de los movilizados entre quienes se integraron a la coalición emergente y aquellos que la resistían señalando los elementos de continuidad con la etapa previa. Las divisiones dificultaron la convergencia en la movilización, como en el caso de las organizaciones piqueteras y de derechos humanos. La polarización creciente a partir de 2008 produjo nuevos impactos, entre estos la división de la CTA y que el conjunto de la protesta social pasase a estar más inscripto en la disputa política general. Pero los principales cuestionamientos al gobierno no vinieron desde la protesta de los sectores populares. El cuestionamiento en las calles no provino tanto de "abajo" como de "arriba" de la estructura social.

El retroceso político-institucional de sectores de las clases dominantes condujo a la emergencia de procesos sociopolíticos basados en la movilización de masas en defensa de sus intereses. Fue en 2008 cuando estos se expresaron por primera vez con nitidez en el denominado "conflicto del campo". Dicho conflicto logró politizar, movilizar y polarizar la sociedad como

11 Esta relevancia se traduce en experiencias de participación. Un estudio por encuesta de carácter estadísticamente representativo de la población del AMBA en 2012 mostraba que el corte de vías de tránsito era la tercera modalidad de acción colectiva (8\%) en lo que refiere al nivel de participación de la población, luego de la movilización (25\%) y la huelga (18\%) (Rebón, 2013). 
ningún otro en la etapa. En marzo de 2008, el gobierno de Cristina Fernández de Kirchner, con el objeto de resolver un problema fiscal en ciernes, elevó la alícuota de retención a la exportación de diversos granos, en especial la soja, y vinculó su variación a la del precio internacional de los productos (Kulfas, 2016). En un contexto de elevada rentabilidad en la exportación de granos y en el marco de una cosecha récord pronta a ser levantada, la medida fue vivida por los propietarios rurales como la confiscación de una ganancia planificada. Las principales entidades corporativas del sector se unificaron en reclamo de la derogación de la medida. Esto incluyó tanto a organizaciones de la pequeña producción rural - como la Federación Agraria, con una rica historia de alianza con los movimientos populares - como a la más tradicional representante de los terratenientes, la Sociedad Rural.

La "mesa de enlace" conformada por las entidades declaró la no comercialización de granos ni carne, estableciendo verdaderos controles de las rutas - "cortes" - para garantizar la efectivización de la medida. Se inició así un intenso conflicto que tomó desprevenido al gobierno y configuró un movimiento social (Tilly, 2008) que, a semejanza de los desarrollados en los sectores populares en el período, centró su movilización en la acción directa - cortes y escraches, por ejemplo - y la práctica asamblearia. La movilización de masas unificó a los distintos estratos de la propiedad rural tras un proyecto hegemonizado por los sectores más concentrados del capital rural. Pero el conflicto excedió lo agrario, movilizando a la oposición social y política, especialmente a aquellos sectores que se sentían disconformes por las medidas reformistas del gobierno. En un país urbano desde larga data, la lucha contra las retenciones involucró y alineó al conjunto de la población, conformando una dualidad de poder que paralizó el país y desabasteció a los principales centros urbanos, y expresó el más intenso proceso de generalización de la acción directa poscrisis de 2001 (Antón, et al., 2011). La magnitud del conflicto llevó a que en ese año se alcanzara, según el relevamiento del Centro de Estudios para la Nueva Mayoría (2016), el nivel más alto de cortes entre 2001 y 2015 - el $26 \%$ de todo el período - y a que, según datos del Programa de Investigación del Movimiento de la Sociedad Argentina, los propietarios desplazaran a los trabajadores ocupados como componente principal de la protesta $-31 \%$ frente a $25 \%$, respectivamente- (Antón, et al., 2011).

Cada fuerza cortó transversalmente la pirámide social con diferentes composiciones. Mientras que las movilizaciones y cacerolazos del "campo" tuvieron su base social en las capas medias y altas, el gobierno basó su movilización en los trabajadores organizados gremialmente y en los pobres urbanos, junto a grupos de intelectuales progresistas. Finalmente, ante la prolongación del conflicto, el gobierno solicitó la aprobación de la medida por 
el Parlamento, introduciendo algunas reformas. La medida fue rechazada en el Senado, donde el propio vicepresidente votó en contra. Un año después, el gobierno vio marcadamente disminuido su caudal electoral en las elecciones legislativas, al perder la mayoría parlamentaria. Pero, lejos de significar la derrota estratégica de su política reformista, el gobierno radicalizó su agenda con el objetivo de consolidar su fuerza. En este marco, desarrolló sus medidas más audaces, entre ellas la estatización de los fondos jubilatorios y de empresas privatizadas en los noventa, una Ley de Servicios Audiovisuales de carácter antimonopólico - en el marco de un conflicto con el principal grupo de medios - y la extensión de la protección social a los sectores más excluidos con la asignación universal por hijo. El conflicto dejó configurada con nitidez una situación de polarización política, con tendencias antagonistas en su seno.

El segundo gran momento de las movilizaciones de "arriba" fue el ciclo de cacerolazos que surgió en 2012, luego de la contundente reelección, en el año precedente, de Fernández de Kirchner con más del 54\% de los votos. Las protestas antagonizaron con el gobierno y tuvieron sus expresiones más masivas el 13 de septiembre y el 8 de noviembre de 2012 - la más multitudinaria de todas - y el 18 de abril de 2013. La convocatoria se organizó a partir de grupos virtuales antikirchneristas, autodefinidos como "apartidarios", y fue replicada y amplificada por los medios de comunicación enfrentados con el gobierno. La movilización fue presentada públicamente como "espontánea y ciudadana", contrastando en términos simbólicos con la movilización popular representada como vinculada al clientelismo del Estado. El uso del cacerolazo y el discurso "apolítico" dieron cuenta de la huella cultural de los tiempos de crisis. La estructura de movilización presentó un carácter individualizado y de pequeños grupos informales, sin presencia pública de colectivos organizados. No obstante, con el desarrollo del ciclo emergió la presencia de grupos organizados y partidarios (De Piero y Gradin, 2015). A diferencia de lo ocurrido en el conflicto del campo, no hubo un reclamo específico que articulara las movilizaciones: cada una desarrolló un abanico de demandas expresado por sus participantes, que fue variando entre elementos tales como la oposición al cambio de la Constitución para habilitar una nueva reelección presidencial, la inflación, los discursos de la presidenta en cadena nacional, los controles a la compra de divisa, la reforma judicial, la corrupción, entre muchos otros.

Lo que sí articuló la diversidad de la protesta fue su oposición en términos antagónicos al gobierno nacional. Se expresó una suerte de cadena equivalencial negativa que unificó a los agraviados por compartir la fuente del malestar. A diferencia de los cacerolazos de 2001, las movilizaciones no se produjeron en la base social del gobierno; por el contrario, a pesar de su 
masividad, no lograron trascender el núcleo duro de la oposición social al gobierno con su eje en las capas medio-altas de la población y tampoco vincularse con sectores que se desgajaron de la alianza del gobierno, como los de origen sindical ya referidos (Gamallo, 2012). No conformaron tampoco un movimiento social en un sentido pleno, dada la ausencia de núcleos organizativos públicos de coordinación. Su impacto es difícil de evaluar, dadas la heterogeneidad y ambigüedad de sus metas.

El gobierno mostró respuestas parciales a algunas de ellas, como por ejemplo medidas contra la inflación o la inseguridad, o la flexibilización del control de divisas. Pero no es nítido que estas movilizaciones hayan sido lo determinante en estas medidas, su impacto es en todo caso indirecto (De Piero y Gradin, 2015). En nuestra hipótesis, su rol central fue el de mantener movilizada a la oposición social al gobierno y desgastar su triunfo electoral obstaculizando la posible consolidación de un proceso hegemónico, dadas las condiciones de debilidad de la oposición política. Posteriormente, la marcha convocada por un grupo de fiscales en febrero de 2015 buscó activar al mismo sector, en este caso pidiendo justicia ante la muerte del fiscal Alberto Nisman y acusando al gobierno de participar en su supuesto asesinato. Esta vez, si bien la movilización fue masiva, tuvo las mismas limitaciones sociales que las anteriores y no logró replicarse. El calendario electoral presidencial corrió el eje, ya definitivamente, de la oposición social a la política.

Como hemos descripto, la etapa se caracterizó por un impacto político divergente de la protesta social. Más allá de las condiciones favorables para la lucha corporativa de diversos sectores populares y progresistas, en nuestra hipótesis, no fue la protesta la fuente directa de las principales reformas planteadas por el gobierno, sobre todo luego de su etapa inicial y con nitidez a partir de 2008. Esto es así por dos razones. En primer lugar, porque, una vez que la coalición política se consolidó, muchas de las demandas fueron planteadas en su interior por parte de las organizaciones a través de mecanismos más cercanos a la lógica del grupo de interés que a la de la protesta social. Pero, en segundo lugar, porque con su estilo centralizado de conducción el gobierno avanzó en la agenda reformista más allá incluso de que existiera como demanda de la sociedad civil ${ }^{12}$. De este modo, medidas de la agenda reformista, como las nacionalizaciones o la asignación universal por hijo,

12 En sectores tanto del activismo político como del campo intelectual se planteó durante estos años la discusión de si la posición política del gobierno era más "antineoliberal" que la de la sociedad civil (Danani, et al., 2012). En nuestra hipótesis, el gobierno avanzó pragmáticamente resolviendo problemas apoyado en un consenso pasivo favorable a muchas de las medidas. Sin embargo, en algunas áreas su impronta fue más crítica que la dominante (conflicto del campo, por ejemplo) y en otras, decididamente menos rupturista, como en el área socioambiental (Svampa, 2017) o en la organización del servicio de trenes, al menos hasta la parte final del mandato (Pérez y Rebón, 2017). 
entre otras, no fueron el resultado de la correlación de fuerza de la sociedad civil, de masas en las calles reclamándolas, sino el resultado de decisiones políticas que fueron legitimadas públicamente a posteriori. En todo caso el rol de la protesta social fue indirecto y diferido en su traslación a la agenda de gobierno. En muchos casos se trató de demandas y luchas de larga data de las organizaciones sociales, que fueron retomadas fuera del calendario de la protesta por el gobierno, que definió los tiempos y las formas de su implementación, y también las capitalizó políticamente.

El rol político central provino de la protesta de "arriba". Esta tiene más un rol negativo de obstrucción, veto y control, de generar condiciones de dificultad para la aplicación de políticas, que de logro de medidas positivas. En todo caso, la política positiva fue la instalación de temas en la agenda pública, para algunos de los cuales el gobierno formuló respuestas parciales, pero que, sobre todo, nutrieron la agenda de la oposición. Los puntos máximos de este proceso se alcanzaron en dos años no electorales con fuerte debilidad de la oposición política, 2008 y 2012, cuando las movilizaciones fueron las más masivas que se registraron. En 2015, el ciclo político tuvo su cierre a partir de la confrontación electoral, con el triunfo en un ajustado balotaje de Mauricio Macri (PRO-Cambiemos), el exponente político más antagónico al proyecto kirchnerista. A pesar de esto, la confrontación se resolvió agonísticamente (Mouffe, 2011) y la transición política se produjo sin sobresaltos, abriendo una nueva etapa.

\section{En tiempos de Cambiemos}

A pesar de su moderado discurso de campaña, el gobierno de la alianza Cambiemos procuró tempranamente traducir su triunfo electoral en una reestructuración societal. Desde una orientación neoliberal, en lo económico planteó una nueva fase de apertura, liberalización de la economía, endeudamiento y transferencia de recursos, a través de diversos mecanismos, a sectores concentrados de la economía (financiero, agrario y energético) a expensas del conjunto social (Ferrer, 2016). Su programa de gobierno implica desandar diversas regulaciones y derechos sociales instalados en la década previa, o incluso previamente, y alterar la distribución del poder estructural en la sociedad argentina, tanto en el interior de la clase dominante, donde el capital financiero gana fuerte peso, como en las relaciones entre el capital y el trabajo (Basualdo y Manzanelli, 2017).

En este último sentido, plantea como uno de sus ejes centrales la búsqueda del disciplinamiento para los trabajadores y los movimientos populares. Su parentesco con las políticas de la década de los noventa no puede soslayar su originalidad. Para comenzar, se trata del primer gobierno constituido por la élite de la clase dominante que arribó al gobierno en elecciones 
competitivas y con la unificación, de modo inédito, de las élites económica, política, judicial y mediática, lo cual le otorga una significativa acumulación de poder social a pesar de no tener mayoría parlamentaria ni presencia importante en los sectores populares de la sociedad civil. En segundo lugar, la novedad estriba en que las clases subalternas, en términos generales, durante el siglo XXI han enriquecido su cultura de lucha, incrementado su poder institucional y alcanzado nuevos derechos. Además, a diferencia de lo ocurrido en los noventa, el modelo de acumulación y de regulación social previo, a pesar de las evidentes tensiones y dificultades de los últimos años, no había entrado en una crisis general como para justificar socialmente un cambio radical (Pierbattisti, 2017). En estas condiciones, la determinación de reestructuración del capitalismo argentino está encontrando uno de sus obstáculos más significativos en la protesta social. De este modo, el avance de las contrarreformas está siendo regulado pragmáticamente, retrocediendo en ocasiones, prolongando e incluso profundizando algunas políticas sociales previas, y avanzando allí donde las líneas de defensa son más débiles y no desatan procesos que vulneran la política general.

Las protestas de los trabajadores en sus distintas expresiones configuran el eje principal de la resistencia. Esta se caracteriza por la diversidad en sus componentes, por su masividad (Varela, 2017) y por su capacidad puntual de convergencia táctica. Según datos del Ministerio de Trabajo (2017), durante el primer año de este gobierno en el campo del conflicto laboral se alcanzó la mayor cantidad de huelguistas desde que comenzó la serie, en 2006, con el escalamiento de reclamos netamente defensivos, como los reclamos por despidos. Al igual que en diversos momentos de reestructuración regresiva de las condiciones para el trabajo, la dualidad estructural del actor sindical se expresa en la tensión entre la resistencia y la integración ${ }^{13}$.

Para la parte dominante del sindicalismo, la política está pasando por el nivel de la negociación con el objeto de preservar recursos de las organizaciones y sus conducciones y moderar o administrar el impacto de las reformas en los sectores que representan. Para otros actores del sindicalismo, como los enrolados en las CTA y muchos de ellos en la CGT, la búsqueda está siendo expresar la disconformidad y enfrentar abiertamente la política de gobierno. Sin embargo, el avance de la apertura de las importaciones, la disminución del salario real, el aumento de los despidos, el horizonte de reformas planteado y las avanzadas políticas y judiciales sobre el sindicalismo, junto al

13 Con dualidad estructural nos referimos a que el actor sindical, por una parte, expresa el malestar y las demandas del trabajo, configurando una lógica de la movilización, y, por otra, se muestra como un actor en el sistema que administra y controla la disconformidad, garantizando estabilidad en los recursos, configurando una lógica del grupo de interés. Ambos polos presentes y necesarios al actor sindical originan distintos tipos de sindicalismo, según sea su peso relativo en la práctica (Offe y Wiesenthal, 1992). 
malestar en las bases, están configurando la construcción de momentos de masiva movilización unitaria y modificando recurrentemente las fronteras entre los sectores más vinculados a uno u otro eje de orientación. A diferencia de los noventa, estos momentos de unidad incorporaron desde el inicio a expresiones organizativas de los sectores más pauperizados y precarios del trabajo, como trabajadores de cooperativas, pequeños emprendimientos, vendedores ambulantes, entre otros ${ }^{14}$. Estos actores gremiales, que expresan en el campo organizativo la persistencia estructural de fuerte desigualdades en el interior de la fuerza de trabajo, mostraron una gran capacidad de movilización y de articulación con los actores sindicales, al obtener en un contexto desfavorable la promulgación de la ley de emergencia económica que, entre otros elementos, implicó la formulación de un salario complementario para trabajadores de la economía popular (Abal Medina, 2017).

Otro componente significativo de la protesta es el movimiento de derechos humanos. Este movimiento se está articulando crecientemente en oposición a cambios en la política de memoria y justicia respecto a los crímenes de la última dictadura cívico-militar. El principal acontecimiento en este campo ocurrió en mayo de 2017, cuando la Corte Suprema de Justicia de la Nación, luego de la incorporación de dos nuevos jueces propuestos por el presidente, falló a favor de que los condenados por delitos de lesa humanidad pudieran computar doble el tiempo que estuvieron detenidos antes de contar con una sentencia firme. Esto, en términos prácticos, implicaba la liberación de una parte significativa de los condenados. El fallo despertó una ola de repudio, las organizaciones de derechos humanos convocaron a movilizaciones en todo el país que representaron el principal hecho de masas del período. El gobierno, luego de declaraciones iniciales que legitimaban su aplicación, ante el malestar social cambió de posición y participó en la rápida sanción de una ley que excluye la aplicación del "dos por uno" en los delitos de lesa humanidad. El fallo quedó finalmente suspendido en sus efectos prácticos. En el período, las organizaciones están manteniendo importantes niveles de movilización e integrando cada vez más en su agenda la lucha contra la política represiva del gobierno.

Finalmente, el renovado movimiento de género, surgido a mediados de 2015 bajo la consigna Ni una menos y que logró instalar en el país - con repercusiones en otros países de Latinoamérica - la violencia y la desigualdad de género como problema público, mantiene una activa movilización en el período (Cabral y Acacio, 2016). El movimiento produjo un proceso

14 El desarrollo de la Confederación de los Trabajadores de la Economía Popular y otras organizaciones expresó la capacidad de un sector del movimiento piquetero de articular con otros actores, reestructurando su acción hacia el territorio socioproductivo en convergencia y tensión con la política social del gobierno anterior que alentó la formación de cooperativas y emprendimientos comunitarios (Abal Medina, 2017; Kasparian, 2017). 
de politización de sus demandas contra el gobierno nacional por recortes de presupuesto y falta de implementación de políticas vinculadas a la temática de género, la represión de movilizaciones e incluso la política económica y social en general. También, como parte de la actividad del movimiento, se consolidó públicamente la instalación del reclamo del derecho al aborto legal, seguro y gratuito, configurando las condiciones para su posterior desarrollo - más allá del período abarcado por este trabajo - en un intenso y masivo movimiento social.

Pocos momentos de la historia sintetizan con nitidez un ciclo de movilización como marzo de 2017. En esos días se registraron masivas protestas de distintos sectores. Cinco movilizaciones merecen destacarse por su carácter multitudinario. Cada una de ellas convocó a centenares de miles. Entre estas se encuentran la marcha federal docente en el marco de una prolongada huelga contra el desconocimiento de los mecanismos paritarios institucionalizados durante el período anterior, el acto de protesta convocado por la CGT con la adhesión de la CTA y organizaciones sociales que terminó en incidentes ante la falta de precisión de la conducción en torno a la convocatoria a un paro general, una marcha y paro de Ni una menos en reclamo de igualdad de género y la movilización del 24 de marzo en conmemoración del Día de la Memoria y contra las declaraciones negacionistas en torno al genocidio de funcionarios gubernamentales. Fue un mes en el que la calle estuvo politizada contra el gobierno como pocas veces en la historia. Pero cuando la iniciativa en el espacio público parecía perdida para este, y a pocos días de materializarse el paro convocado por el conjunto del sindicalismo, surgió desde las redes sociales, con rápida y activa difusión en los medios de comunicación, la convocatoria a una movilización en defensa de la democracia. Esta se materializó el $1^{\circ}$ de abril, sorprendiendo a muchos por su masividad y teniendo como eje la oposición al kirchnerismo y a las movilizaciones reseñadas, y, secundariamente, en apoyo al gobierno. Los cánticos de "no vuelven más", en alusión al gobierno anterior, y "los chicos a la escuela", en alusión al paro docente, dan claridad del contenido de la movilización. La contramarcha mostró que durante el kirchnerismo las capas más acomodadas de la sociedad configuraron una cultura de acción colectiva con sus propias dinámicas y valores. La contramarcha fue menos masiva que buena parte de las movilizaciones opositoras, pero su carácter inesperado y la capacidad mediática de la política de gobierno la convirtieron en una evidencia de su apoyo popular y del hastío hacia la protesta. La acción colectiva, en tanto conflicto no regulado, tiende a resolverse en los términos de su percepción social (Mc Adam, et al., 1999). Aun cuando, pocos días después, el primer paro general del sindicalismo en el período alcanzó una gran adhesión - la mayor desde de diciembre de 2001 - , el gobierno encontró impulso para retomar la iniciativa política y avanzar en cambiar las condiciones de la protesta. 
Desde el inicio del gobierno de Macri, dichas condiciones tendieron al cambio. El encarcelamiento de la dirigente Milagro Sala, de la organización social Tupac Amaru, en el marco de un acampe frente a la casa de gobierno de Jujuy, y su posterior mantenimiento en esta situación a partir de la apertura de nuevas causas fue el primer signo evidente del cambio de las condiciones de contorno para la acción colectiva. El uso del sistema judicial contra los integrantes de organizaciones sociales y políticas, una práctica preexistente, adquirió una renovada sistematicidad para el Poder Ejecutivo Nacional y los ejecutivos locales, con el objeto de modificar la correlación de fuerzas en contra de los protagonistas de los reclamos (CELS, 2017) ${ }^{15}$. La avanzada de represión y estigmatización de la protesta adquirió un renovado impulso a partir de abril de 2017. La represión afectó la libertad de reunión y expresión (Amnistía Internacional, 2018). Devino más intensa, incrementándose la cantidad de detenidos (Observatorio de Desarrollo Social, 2017) y alcanzó a sectores y localizaciones que hasta entonces no tendían a ser reprimidos, como por ejemplo maestros que montaban una carpa de protesta en la ciudad de Buenos Aires.

Por otra parte, sectores más habituados a padecer la represión, incluso en la etapa previa (Svampa, 2017), fueron objeto de una avanzada represiva. Este es el caso del pueblo mapuche, cuya lucha por la tierra es objeto de una amplia estigmatización bajo el rótulo de "amenaza terrorista". En este marco, la intervención de fuerzas federales se volvió más recurrente, y se produjeron, en agosto, en el contexto de la represión indiscriminada de un corte de ruta, la desaparición del manifestante Santiago Maldonado - aparecido sin vida más de dos meses después - y, en noviembre, en otra represión a la lucha mapuche, el homicidio del manifestante Rafael Nahuel (Amnistía Internacional, 2018). Desde el Poder Ejecutivo Nacional, las intervenciones y acciones fueron justificadas y reivindicadas, estigmatizando a las víctimas y obstaculizando las investigaciones. Se produjo así un proceso que tiende a cambiar la ecuación entre las formas prescriptas, toleradas y proscriptas de la acción colectiva, a favor de estas últimas. Formas de intervención activa y disruptiva instaladas en el repertorio popular, como los cortes y las tomas, registraron renovados obstáculos a su uso. Pero este proceso no solo afectó la acción directa. La huelga - el derecho a no cooperar como forma de reclamo en la relación laboral - encontró nuevas dificultades ante prácticas antisindi-

15 También se registra una proliferación de causas judiciales contra funcionarios del gobierno anterior, incluida la expresidenta. Estas causas no se restringen a delitos económicos, sino que incorporan, como un nuevo avance de la judicialización de la política ya existente (Nosetto, 2014), causas sobre decisiones de gobierno. Destacan crecientes rasgos de instrumentalización política de los procesos judiciales, entre estos el uso generalizado de la prisión preventiva, el automatismo en la apertura de causas, la selectividad de los avances y su sincronización en términos políticos (Garzón, 2018). 
cales del gobierno y nuevos fallos judiciales (CELS, 2017). La movilización misma - la forma más convencional y generalizada de la protesta -, como veremos a continuación, está siendo objeto de una creciente práctica represiva.

Los acontecimientos de diciembre de 2017 muestran con nitidez este nuevo contexto. Luego de su triunfo en las elecciones legislativas de medio término, en octubre, el gobierno redobló su apuesta de contrarreforma, que tiene como ejes, entre otros ámbitos, el terreno laboral, el previsional y el impositivo. La reforma previsional, que consistió básicamente en el cambio del índice de actualización de las jubilaciones - implicando una significativa disminución inicial del aumento jubilatorio-, representó el eje de la resistencia. El día de su tratamiento parlamentario en la Cámara de Diputados, una masiva movilización de sindicatos y organizaciones sociales y políticas en la Plaza del Congreso fue ferozmente reprimida, e incluso diputados fueron objeto de la represión. No obstante, muchas de las columnas de manifestantes, luego de sufrir la represión, se reorganizaron y volvieron a ingresar a la plaza expresando su determinación. Finalmente, ante reclamos de la oposición, luego retomados incluso por sectores del propio oficialismo, la sesión se levantó, sin embargo, la represión y las razias policiales se extendieron por varias horas.

Pocos días después, la amplia oposición social a la reforma se expresó en otra masiva movilización, ante una nueva sesión en el Congreso, que incluyó un paro general de actividades. La concentración derivó rápidamente en enfrentamiento entre las fuerzas de seguridad y centenares de manifestantes que atacaron con palos y piedras las columnas policiales. Los movilizados procuraron permanecer en la plaza a pesar de la represión, hasta que su intensidad logró desalojar el área. Posteriormente, las fuerzas de seguridad practicaron una cacería de manifestantes por el centro de la ciudad con inusitada violencia. Con un saldo, según fuentes oficiales, de 68 detenidos y 162 heridos, entre ellos 88 policías, este representó el episodio de violencia colectiva más importante en el marco de una protesta social desde las jornadas de diciembre de 2001. Pero la represión no apagó la expresión del malestar. Por la noche, Buenos Aires y ciudades del resto del país fueron sacudidas por cacerolazos a través de los que se expresaron protestas contra la reforma del gobierno.

Finalmente, el proyecto, con modificaciones menores, fue sancionado. La reforma avanzó, pero no sin costos para la legitimidad del gobierno, incluso entre sus votantes. También terminó de dañar el principio de acuerdo que existía entre el gobierno y la cúpula de la CGT en torno a la reforma laboral, lo que obligó al gobierno a postergar su debate. El resultado del conflicto muestra que la protesta social en condiciones de debilidad de la oposición 
política es un modo central de canalizar la disconformidad e incluso un dinamizador de la unidad de la oposición política, tal como se pudo registrar en el debate en la Cámara de Diputados (Grimson, 2017). Pero hasta el momento, en líneas generales, la protesta social está imponiendo obstáculos y modificaciones paliativas a las reformas sin poder detener la tendencia del cambio social. Las imágenes de barricadas con humo en el centro de la ciudad de Buenos Aires y de ciudadanos protestando con cacerolas en los barrios rememoran postales de 2001, lo que muestra la vigencia de la protesta como vehículo político ciudadano en la Argentina actual y, también, las dificultades para su contención represiva.

\section{Reflexiones finales}

El análisis precedente nos muestra la persistencia y relevancia de la protesta social en la Argentina de la primera parte del siglo XXI.

La protesta tiene un rol relevante como mecanismo de expresión de demandas hacia el sistema político-institucional. En nuestra hipótesis, su impacto en términos políticos - más allá de lo estrictamente corporativo- es más negativo que positivo y su principal rol es el veto fáctico de acciones de gobierno o, en caso extremo, del gobierno mismo. Ante la debilidad del sistema de partidos en el período de análisis, en particular de la oposición política, la protesta se configura como forma privilegiada de oposición social en diversos momentos ${ }^{16}$. Este carácter conduce reiteradamente a que los ciclos masivos de protesta se nutran en su composición de las posiciones en la estructura social que tienden a no formar parte de la base de la alianza de gobierno.

En 2001, el carácter destituyente adquirió tal intensidad, precisamente porque una parte sustantiva de la base social del gobierno se movilizó en su contra. Por otra parte, la predisposición a la protesta en la población hace que se convierta en un relevante horizonte de restricción de la política pública. Como hemos visto, la protesta como horizonte incide significativamente en decisiones de gobierno en campos tan diversos como la política económica, social o represiva. Finalmente, en casos como las movilizaciones en apoyo al gobierno en el conflicto del campo o frente a las marchas opositoras en 2017, opera negativamente contra aquellos que se movilizan - contraprotesta-, lo que afirma la acción de gobierno.

16 El carácter negativo es un facilitador de la acción colectiva. La defensa permite definir con nitidez el adversario con base en agravios al afectar posiciones previamente legitimadas (Thompson, 1979) y promueve la convergencia de actores diversos en sus metas atenuando posibles controversias (Rosanvallon, 2007). La selección de la defensa como estrategia de acción nace de la debilidad en el campo de relaciones (Clausewitz, 1984), por esta razón el remanido debate entre los activistas de base acerca de la necesidad de pasar de lo "negativo" a lo "propositivo" en la lucha no deja de ser, en muchos casos, más que una expresión de deseo. 
Secundariamente, la protesta incide de manera positiva en las medidas de gobierno, básicamente a través de dos formas. En primer lugar, la más clásica, a partir de la instalación y el logro de una política, en general mediante apoyos políticos transversales, es el caso, por ejemplo, de la Ley de Emergencia Social durante el gobierno de Macri o la Ley de Matrimonio Igualitario durante el kirchnerismo (Figari, 2011). En segundo lugar, incide indirectamente, como elemento configurador de temas en la agenda pública que a posteriori puedan ser incorporados por iniciativa del gobierno en su campo de acción, como, por ejemplo, la asignación universal por hijo. Profundizar en el conjunto de hipótesis aquí esbozadas requiere de una futura investigación empírica.

Por otra parte, la práctica generalizada de la acción colectiva abre desafíos investigativos a los estudios en la temática. En primer lugar, el de conocer en qué medida se ha ampliado en el siglo XXI la proporción de población que participa en la acción colectiva, así como situar dichos niveles en comparación con otros países. A pesar de la abundancia de estudios sobre la acción colectiva, carecemos de registros sistemáticos a nivel nacional sobre la experiencia de la población, más allá de algunos avances puntuales sobre el AMBA (Gómez, 2008; Rebón, 2013). En segundo lugar, en sintonía con lo ocurrido en otros países (Fillieule y Tartakowsky, 2015), se registra una mayor heterogeneidad de los participantes, la protesta no es patrimonio de ningún grupo social. La presencia de culturas de lucha diversas -e incluso contrapuestas - expresa procesos de sedimentación que retoman y actualizan las luchas del pasado. Estudiar sus repertorios, estructuras de movilización e identidades de modo comparado es, sin lugar a duda, un desafío pendiente.

Como hemos descripto, el período de análisis presenta cambios en la caja de herramientas de lucha de distintas identidades. En los tiempos de crisis se consolidó un nuevo repertorio de acción en el cual las formas disruptivas de la protesta alcanzaron alta relevancia. Durante la etapa kirchnerista, a pesar de que se produce una institucionalización del conflicto, este carácter disruptivo persistió. En el período actual se cambian las condiciones de la acción colectiva con el avance de formas que en la práctica habían entrado relativamente en un umbral de tolerancia fáctica hacia un terreno cada vez más nítidamente de proscripción. Los procesos represivos amenazan incluso con alcanzar las formas más convencionales y clásicas de la protesta.

La política de gobierno, crecientemente antagónica - exclusión del oponente - , tiende a plantear un conjunto de desafíos para las organizaciones civiles y sociales. En primer lugar, la necesidad de incorporar a su agenda de reclamo y marco de alianza la defensa de las garantías democráticas e invertir importantes recursos organizativos para enfrentar los procesos de judicialización. En segundo lugar, actualizar las culturas de acción a las nuevas 
condiciones. El cambio de condiciones conduce a las organizaciones a recuperar el rico acervo de medidas de seguridad de otros momentos de la historia y actualizarlo a las nuevas condiciones tecnológicas. Finalmente, como lo demuestra la investigación comparada sobre la acción colectiva (Tilly, 2008), el cierre de las condiciones de la protesta tiende a conducir a su expresión en términos de violencia colectiva. La reiteración de incidentes en diversas movilizaciones en el último tiempo plantea a las organizaciones el desafío de mayores medidas de coordinación y autocontrol y a practicar sistemáticamente estrategias de no violencia activa que, sin ceder en términos de movilización y disrupción, no incurran en el combate violento - recurrentemente desigual - con las fuerzas de seguridad, para evitar así la profundización de procesos de deslegitimación y criminalización de los reclamos. La fuerza moral es uno de los elementos más poderosos de la protesta de la sociedad civil (Ameglio, 2002). Protegerla y fortalecerla en la acción colectiva supone recuperar y actualizar el acervo existente en las historias de las luchas sociales para enriquecer las estrategias del presente.

Para concluir, remarquemos: la política en las calles es uno de los mecanismos a través de los cuales se expresa la discusión por la direccionalidad del país. En los tiempos de la crisis de 2001 fue la expresión de la crisis de la hegemonía de la valorización financiera y el cuestionamiento a las personificaciones dominantes de la política desde múltiples posiciones de la estructura social y diversidad de perspectivas. A partir de 2003 tendió a tener contenidos sociales y políticos más definidos. Un gobierno reformista que alteró parte de las tendencias dominantes desató un proceso de polarización que atravesó la acción colectiva. El arribo en 2015 de un gobierno de tendencia contraria parecería reeditar las tesis del "empate hegemónico" (Portantiero, 2003) entre distintos proyectos de capitalismo para el país, que, entre otros tópicos, implica la distribución del poder social entre las clases subalternas. La pendularidad de ciclos (Ferrer, 2016) sugiere la existencia de un bloqueo mutuo. Sin embargo, vale la pena distinguir que los términos no son iguales - no hay polaridad - para las fuerzas en disputa. Las moderadas reversiones de las reformas liberales de los noventa, obtenidas lentamente durante el ciclo anterior, en muchos casos, son actualmente revertidas con rapidez. Pero la direccionalidad no está resuelta ni consolidada. Realizar la victoria en términos de la élite dominante, resolver los términos del empate, presupone la derrota social de la resistencia popular. Para esta, la defensa de las condiciones alcanzadas y del marco de garantías institucionales es el prerrequisito para luchar a futuro por ampliar los horizontes de igualación. Para todos, independientemente de los términos en los que lo expresen, la lucha continúa. 


\section{Referencias bibliográficas}

Abal Medina, P. (2017). Los movimientos obreros organizados de Argentina (2003-2015). En Abal Medina, A. Natalucci y F. Rosso. ¿Existe la clase obrera? Buenos Aires: Capital Intelectual, pp. 21-61.

Ameglio, P. (2002). Gandhi y la desobediencia civil: México hoy. México: Plaza y Valdés. Amnistía Internacional (2018). Informe 2017-2018. La situación de los derechos humanos en el mundo. Disponible en: <https://www.amnesty.org/es/latest/research/2018/02/ annual-report-201718/> [acceso 20-2-2018].

Antón, G.; J. Castro; J. Rebón y R. Salgado (2011). Una década en disputa. Apuntes sobre las luchas sociales en Argentina. En: M. Modonessi y J. Rebón. Una década en movimiento. Luchas populares en América Latina en el amanecer del siglo XXI. Buenos Aires: Prometeo-CLACSO-UBA, pp. 19-44.

Auyero, J. (2002). Los cambios en el repertorio de la protesta social en la Argentina. Desarrollo económico. 42(166), pp. 187-210.

Auyero, J. (2007). La zona gris. Violencia colectiva y política partidaria en la Argentina contemporánea. Buenos Aires: Siglo XXI.

Basualdo, E. (2013). Estudios de historia económica argentina desde mediados del siglo XX a la actualidad. Buenos Aires: Siglo XXI.

Basualdo, E. y P. Manzanelli (2017). La deuda externa de Macri en perspectiva histórica. Voces en el Fénix, 64, pp. 19-25.

Cabral, P. y Acacio, J. (2016). La violencia de género como problema público. Las movilizaciones por "Ni una menos" en la Argentina. Questión. 1(51), pp. 170-187.

CELS (2017). Derechos humanos en la argentina. Informe 2017. Buenos Aires: CELSSiglo XXI.

Centro de Estudios para la Nueva Mayoría (2016). Los cortes de rutas y vías públicas (19972016). Disponible en: <http://www.nuevamayoria.com/index2.php?option=com_ content\&do_pdf=1\&id=5037> [acceso 20-2-2018].

Cheresky, I. (2011). Representación política y contra/democracia. Ciencias Sociales, 78, pp. 30-37.

Clausewitz, C. (1984). De la guerra. Barcelona: Editorial Labor.

Danani, C.; M. Gómez; M. Kessler y F. Schuster (2012). Participación, régimen político y movimientos sociales. ¿Hacia una ciudadanía más inclusiva? Argumentos. Revista de crítica social, 14, pp. 1-40.

De Piero, S. y A. Gradin (2015). La sociedad civil "desorganizada". Protestas y oposición en la sociedad civil a los gobiernos kirchneristas. Revista Estado y Políticas Públicas, 5, pp. 19-39.

Etchemendy, S. (2013). La "doble alianza" gobierno-sindicatos en el kirchnerismo (2003-2012). Orígenes, evidencia y perspectivas. En: C. Acuña. Instituciones gubernamentales y actores de la política argentina. Buenos Aires: Siglo XXI, pp. 101-132.

Etchemendy, S. y R. Collier (2008). Golpeados pero de pie. Resurgimiento sindical y neocorporativismo segmentado. Postdata. Revista de reflexión y análisis político, 13, pp. 145-192.

Ferrer, A. (2016). El regreso del neoliberalismo. Le monde diplomatique, Dossier 201, marzo, pp. 4-7. 
Figari, C. (2011). Matrimonio igualitario: ciencia y acción política. En: N. Solari y C. Von Opiela. Matrimonio entre personas del mismo sexo. Ley 26.618-Antecedentes. Implicancias. Efectos. Buenos Aires: La Ley, pp. 101-132.

Fillieule, O. y D. Tartakowsky (2015). La manifestación. Cuando la acción colectiva toma las calles. Buenos Aires: Siglo XXI.

Gamallo, L. (2012). Entre paros y cacerolazos: Apuntes sobre la conflictividad social en la Argentina reciente. Anuari del Conflicte Social, 2, pp. 877-908.

Garzón, B. (2018). La instrumentalización de la justicia. Página 12, [en línea] 6 de febrero. Disponible en: <https:/www.pagina12.com.ar/93920-la-instrumentalizacion-de-lajusticia> [acceso 20/2/2018].

Gómez, M. (2008). Una caracterización de la predisposición a la acción colectiva y la participación pos 2001 en la Argentina. Resultados de una encuesta. En: CD-ROM Los movimientos sociales en América Latina. Pasado, presente y perspectivas. 1, pp. 640-655.

Gómez, M. (2009). La acción colectiva sindical y la recomposición de la respuesta política estatal en la Argentina 2003-2007. En: J. Neffa, E. de la Garza y L. Muñiz Terra (comps.). El mundo del trabajo en América Latina. Permanencia, diversidad y cambios. Buenos Aires: CLACSO, pp. 161-205.

González, H.; J. Marín; E. Sader; M. Svampa; L. Tapia; M. Maneiro y C. Mera (2010). Los gobiernos progresistas en la región: escenarios futuros. Argumentos. Revista de crítica social, 12, pp. 112-140.

Grimson, A. (2017). Jubilados, violencia y cacerolas ¿Derrota? Revista Anfibia. Disponible en <http://www.revistaanfibia.com/ensayo/derrota/> [acceso 20-2-2018].

Hobsbawm, E. (1997). Historia del siglo XX. 1914-1991. Barcelona, Editorial Grijalbo.

Iñigo Carrera, N. y M. Cotarelo (2006). Génesis y desarrollo de la insurrección espontánea de diciembre de 2001 en Argentina. En: G. Caetano. Sujetos sociales y nuevas formas de protesta en la historia reciente de América Latina. Buenos Aires: CLACSO, pp. 52-99.

Kasparian, D. (2017). De la inducción estatal a la cooperativa sin punteros. El conflicto constituyente en una cooperativa del Programa Argentina Trabaja. Argumentos. Revista de crítica social, 19, pp. 1-42.

Kessler, G. (2014). Controversias sobre la desigualdad: Argentina, 2003-2013. Buenos Aires: Fondo de Cultura Económica.

Kulfas, M. (2016). Los tres kirchnerismos. Una historia de la economía argentina. 20032015. Buenos Aires: Siglo XXI.

La Vaca (2011). Los muertos del 19/20 de diciembre de 2001. Mu, La vaca. [en línea] Disponible en: <http://www.lavaca.org/recuadros/los-muertos-del-1920-de-diciembrede-2001/> [acceso 20-2-2018].

Laclau, E. (2005). La razón populista. Buenos Aires: Fondo de Cultura Económica.

Lobato, M. y J. Suriano (2003). La protesta social en la Argentina. Buenos Aires: Fondo de Cultura Económica.

Maneiro, M. (2012). De encuentros y desencuentros: estado, gobiernos y movimientos de trabajadores desocupados. Buenos Aires: Editorial Biblos.

Marín, J. (2009). Cuaderno 8. Buenos Aires: Colectivo Ediciones/PICASO.

Mc Adam, D.; J. Mc Carthy y M. Zald (1999). Movimientos sociales: perspectivas comparadas. Oportunidades políticas, estructuras de movilización y marcos interpretativos culturales. Madrid: Istmo.

Merino, G. (2012). El movimiento obrero organizado, la crisis de 2001 y el gobierno de Duhalde. Sociohistórica / Cuadernos del CISH, 30, pp. 87-119. 
Ministerio de Trabajo, Empleo y Seguridad Social (2016). La conflictividad laboral durante el año 2016. Disponible en: <http://www.trabajo.gob.ar/left/estadisticas/ descargas/ConLab/Conflicto_Laboral_2017T2.pdf> [acceso 20-2-2018].

Moscovich, L.; A. Santella; P. Semán; J. Rebón, L. Gamallo y M. Maneiro (2017). La conflictividad social en Argentina en el siglo XXI. Argumentos. Revista de crítica social, 19, pp. 1-42.

Mouffe, Ch. (2011). En torno a lo político. México: Fondo de Cultura Económica.

Natalucci, A. (2017). El sindicalismo peronista durante el kirchnerismo (2003-2015). En P. Abal Medina, A. Natalucci y F. Rosso ¿Existe la clase obrera? Buenos Aires: Capital Intelectual, pp. 63-123.

Nosetto, L. (2014). Reflexiones teóricas sobre la judicialización de la política argentina, DAAPGE, 23, pp. 93-123.

Observatorio del Derecho Social (2017). Informe anual [en línea]. Disponible en: <http:// www.obderechosocial.org.ar/docs/anual_2017.pdf> [acceso 20/2/2018].

Offe, C. y H. Wiesenthal (1992). Dos lógicas de la acción colectiva. La gestión política. Madrid: MTySS.

Palomino, H. y P. Dalle (2016). Movilización, cambios en la estructura de clases y convergencia de ingresos en Argentina entre 2003 y 2013. Desarrollo Económico, 56(218), pp. 59-100.

Pereyra, S.; G. Pérez y F. Schuster (2017). Tendencias de la protesta social en Argentina, 1989-2007. En: P. Almeida y A. Cordero. Movimientos sociales en América Latina. Perspectivas, tendencias y casos. Buenos Aires: CLACSO, pp. 577-618.

Pérez, V. y J. Rebón (2012). La perturbación como motor de la historia. Los ferrocarriles metropolitanos durante el kirchnerismo. Buenos Aires: Editorial Biblos.

Pérez, V. y J. Rebón (2012). Las vías de la acción directa. Buenos Aires: Aurelia Rivera libros.

Pérez, V. y J. Rebón (2016). El retorno del Estado. Valoraciones sociales en torno a las empresas estatales. Estudios Sociales, 50(1), pp. 77-104.

Pérez, V. y J. Rebón, coords. (2017). La perturbación como motor de la historia. Los ferrocarriles metropolitanos durante el kirchnerismo. Buenos Aires: Editorial Biblos.

Pierbattisti, D. (2017). Diciembre de 2015. Bordes. Revista de Política, Derecho y Sociedad. [en línea] Disponible en: <http://revistabordes.com.ar/diciembre-de-2015/> [acceso 20-2-2018].

Piva, A. (2015). Economía y política en la Argentina kirchnerista. Buenos Aires: Batalla de ideas.

Polanyi, K. (2001). The Great Transformation: the Political and Economic Origins of Our Time. Boston: Beacon Press. [1944].

Portantiero, J. (2003). Clases dominantes y crisis política en la Argentina actual. [e-book]. Buenos Aires: Editorial Del Cardo. Disponible en: Biblioteca Virtual Universal $<$ http://www.biblioteca.org.ar/libros/656182.pdf> [acceso 20-2-2018].

Pucciarelli, A. y A. Castellani (2014). Los años de la Alianza. La crisis del orden neoliberal. Buenos Aires: Siglo XXI.

Rebón, J. (2007). La empresa de la autonomía. Trabajadores recuperando la producción. Buenos Aires: Colectivo Ediciones/ PICASO.

Rebón, J. (2013). La legitimidad social de las formas de lucha. Una aproximación a partir de la investigación por encuesta. Observatorio Social de América Latina, 36, pp. 193214.

Rebón, J. y C. Hernández (2017). Las formas de la acción colectiva en el sistema ferroviario de pasajeros de la Región Metropolitana de Buenos Aires. En: Revista 
Transporte y Territorio, 16, pp. 201-219. Disponible en: <http://revistascientificas. filo.uba.ar/index.php/rtt/article/view/3610/3305> [acceso 20/2/2018].

Rosanvallon P. (2007). La contrademocracia: La politica en la era de la desconfianza. Buenos Aires: Ed. Manantial.

Schuster, F. (2011). Dos días que cambiaron el futuro. Ciencias Sociales, 79, pp. 30-37.

Silver, B. (2005). Fuerzas del trabajo. Los movimientos obreros y la globalización desde 1880. Madrid: Akal.

Spaltemberg, R. (2012). La diversidad de los conflictos laborales: dispersión y centralización en las lógicas de acción de los asalariados privados. Trabajo, ocupación y empleo, 12 , pp. 37-60.

Svampa, M. (2017). Del cambio de época al fin de ciclo. Buenos Aires: Edhasa.

Tapia, L. (2008). Política salvaje. La Paz: CLACSO, Muela del Diablo, Comunas.

Thompson, E. (1979). La economía moral de la multitud. En: E. Thompson. Tradición, revuelta y consciencia de clase. Barcelona: Editorial Crítica, pp. 62-134.

Tilly, Ch. (2008). Contentious performances. Nueva York: Cambridge University Press.

Varela, P. (2017). La conflictividad laboral durante el primer año de Macri. ¿Quién resiste? Ciencias Sociales, 93, pp. 18-21.

Wright, E. (2000). Workers power, Capitalist interests and class compromise. American Journal of Sociology, 105(4), pp. 957-1002. 